*In memory of great collector, entomologist and geologist from Krasnoyarsk City Igor Semenovich Zakharzhevsky (1924-2019)

\title{
New records of Heterocera (Insecta, Lepidoptera) from the Republic of Khakassia and South of Krasnoyarsk region (South Siberia, Russia)
}

\author{
R. E. Maksimov' , M. A. Ivanov², S. A. Knyazev³. \\ ${ }^{1}$ Karl Marx St. 19, app.5, Abakan, Khakassia Rep., Russia, 655017 \\ E-mail: riverabakan@mail.ru \\ 22-nd Khabarovskaya St. 1, app. 36, Krasnoyarsk, Russia, 660041 \\ E-mail:ima54@mail.ru \\ ${ }^{3}$ Russian Entomological Society, Irtyshskaya Naberezhnaya St, 14-16, Omsk, Russia, 644042 \\ ${ }^{3}$ Altai State University, Lenina St. 61, Barnaul, Russia, 656049 \\ E-mail:konungomsk@yandex.ru
}

\begin{abstract}
Here we presented the new data and new records of Lepidoptera (Crambidae, Cossidae, Lasiocampidae, Drepanidae, Geometridae, Notodontidae, Noctuidae s.l., Arctiidae) from the Khakassia Republic and the South of Krasnoyarsk region. A total of 147 species are reported for the fauna of both regions for the first time, based on the literature records and personal data collected in 1999-2019. We collected the moths in Khakassia and South of Krasnoyarsk region in more than 40 localities, from the steppe zone to the alpine area of the West Sayan, the Kuznetsky Alatau, and foothills of East Sayan Mountains. Specimens were collected in the daylight by standard methods using butterfly net and in the night, using mercury lamp Sylvania (250W) and inverter generator (1000W). All collected materials preserved in private collections of R.E. Maksimov in Abakan (RMA), M.A. Ivanov in Krasnoyarsk (MIK), and in private collection of S.A. Knyazev in Omsk (SKO). The presence of several species have been considered doubtful before our study. We suggested that the regional list of Heterocera of Khakassia and South of the Krasnoyarsk region should be considerably revised. The great share of the specimens mentioned in this article is presented on the websiite "The Nature of South Siberia" (www.ermak24.com/animalia-insecta.html/).

Key words: Lepidoptera, Heterocera, moths, Siberia, Khakassia, Krasnoyarsk territory, new records.
\end{abstract}

\section{Introduction}

The first information on the Lepidoptera fauna for Khakassia and the South of Krasnoyarsk region was presented bu Yakobson (1901) and Sushkin \& Tshetverikov (1907), who reported 32 butterfly species. This was followed by Meinhard expedition of 1903, the data of which was published later (Meinhard 1912). The notable contribution was made by W. Kozhantshikov (Kozhantshikov 1923; 1925; 1926; 1927; 1928). This time he was a Director of the Minusinsk Martyanov`s Museum and invited to work here a number of qualified lepidopterologists, namely A. Djakonov, N. Filipyev, S. Tshugunov, and E. Wehrly. W. Wnoukovsky clarified and confirmed the identification of many species, worked with A. Meinhard, W. Kozhantchikov and S. Tshugunov old data (Wnoukovsky 1929; 1930).

Much later, Yu. Korshunov and J. Viidalepp (1980; 1982) studied Lepidoptera more detail, but they were limited only for Geometridae family. However, despite the intensive research of Lepidoptera from South Siberia and Altai-Sayan Mountain Country, the revision of species list and study of fauna in Khakassia and South of Krasnoyarsk region was rather episodic and fragmentary. Moreover, the remarkable part of scientific reports was dedicated to butterflies (Lepidoptera, Diurna), while Heterocera fauna is very poorly studied in this region. Some fragmentary information was previously published by Wnoukovsky (1929; 1930), Kozhantshikov (1923; 1925; 1926; 1927), Zolotarenko (1969), Korshunov and Viidalepp (1980; 1982). Thus, these data need to revised. Probably, the main limitations were the inaccessibility of the main part of this territory and lack of modern research equipment.

\section{Material and methods}

All new data has been obtained from our personal collections sampled in 1999-2018. We collected the moths in Khakassia and South of Krasnoyarsk region in more than 40 localities, from the steppe zone to the alpine area of the West Sayan, the Kuznetsky Alatau, and foothills of East Sayan Mountains. Specimens were collected in the daylight by standard 
methods using butterfly net and in the night, using mercury lamp Sylvania (250W) and inverter generator (1000W). All collected materials preserved in private collections of R.E. Maksimov in Abakan (RMA), M. A. Ivanov in Krasnoyarsk (MIK), and in private collection of S.A. Knyazev in Omsk (SKO).

The general classification in the check-list accepted by Catalogue of Lepidoptera of Russia (2008) included institutional catalog numbers for specimens preserved in collections.

Localities:

Abakan - Khakassia, near Abakan town, meadow on the river Abakan, 53⒍ $86^{\prime \prime} \mathrm{N}, 91^{\circ} 44^{\prime} 02^{\prime \prime} \mathrm{E}$;

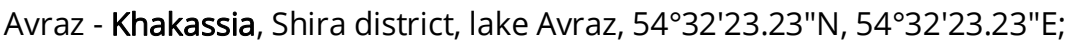

Balankul - Khakassia, near Balankul lake, subtaiga zone, hills \& rocky slope. $53^{\circ} 27^{\prime} 54.85^{\prime \prime} \mathrm{N}, 90^{\circ} 24^{\prime} 18.41^{\prime \prime} \mathrm{E}, \mathrm{h}=850$

m;

Balgash - Krasnoyarsk territory, Krasnoyarsk City vicinities, Balgash boundary, 56 7'30.37"N, 92 ${ }^{\circ} 52^{\prime} 11.10^{\prime \prime} \mathrm{E}$;

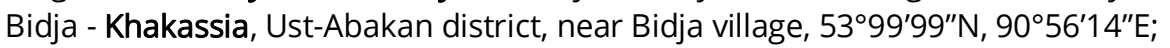

Bograd - Khakassia, Bograd district, near Bograd settlment, 54 ${ }^{\circ} 13^{\prime} 6^{\prime \prime} \mathrm{N}, 90^{\circ} 53^{\prime} 9^{\prime \prime} \mathrm{E}$;

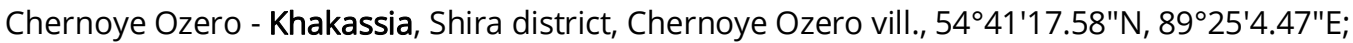

Efremkino - Khakassia, Kuznetsky Alatau, Shira district, near Efremkino, closed marmoreal career, $54^{\circ} 26^{\prime 2} 20.64^{\prime \prime} \mathrm{N}$, $89^{\circ} 26^{\prime} 07.17^{\prime \prime} \mathrm{E}, \mathrm{h}=671 \mathrm{~m}$; $\mathrm{h}=1369 \mathrm{~m}$;

Ergaky - Khakassia, West Sayan, Ergaky ridge, mountain taiga, subalpine meadow, 52 $48^{\prime} 38.83^{\prime \prime} \mathrm{N}, 93^{\circ} 13^{\prime} 51.64^{\prime \prime} \mathrm{E}$,

Galdja - Khakasia, Shira district, near Galdja village, forest-steppe, meadow, 54⒉ $24^{\prime} 23^{\prime \prime} \mathrm{N} 90^{\circ} 30^{\prime} 31^{\prime \prime} \mathrm{E}$;

Gladenkaya - Khakassia, W.Sayan, Gladenkaya mountain, mountain taiga \& tundra, kurumniky, 52 $55^{\prime} 41^{\prime \prime} \mathrm{N}$, $91^{\circ} 23^{\prime} 33^{\prime \prime} \mathrm{E} ;$

Ingol ` - Krasnoyarsk territory, Sharypovo district, Ingol`lake, 55³1'39.65"N, 8850'39.90"E;

Ivanovskie lakes - Khakassia, Kuznetsky Alatau, Ordzenikidze district, subalpine meadows \& tundra, $54^{\circ} 63^{\prime} 88^{\prime \prime} \mathrm{N}$, 8863'16"E;

Karynzul - Krasnoyarsk territory, W. Sayan, M 54 rd., (574km), mountain taiga, on Karynzul stream. $52^{\circ} 59^{\prime} 48.56^{\prime \prime} \mathrm{N}$, $93^{\circ} 12^{\prime} 59.07^{\prime \prime} \mathrm{E}, \mathrm{h}=501 \mathrm{~m}$;

Katcha - Krasnoyarsk territory, Krasnoyarsk vicinities, Katcha rail staition, 56 6 6'2.34"N, 92 ${ }^{\circ} 13^{\prime 2} 22.26 " \mathrm{E}$;

Khudonogovo - Krasnoyarsk territory, Krasnoyarsk vicinities, Khudonogovo village, 56 9'48.46"N, 93¹2'4.22"E;

Krasnoyarsk - Krasnoyarsk territory, Krasnoyarsk City, 56 0'50.75"N, 92²9'59.62"E;

Krasnoye ozero - Khakasia, Ust-Abakan district, steppe near Red Lake, about 53⒊' $\mathrm{N} 91^{\circ} 30^{\prime} \mathrm{E}$;

Lubimoe - Khakassia, W. Sayan, Tashtyp district, Lubimoe lake, alpine meadow, mountain tundra, h=1800 m,

$51^{\circ} 76^{\prime} 63^{\prime \prime} \mathrm{N}, 89^{\circ} 79^{\prime} 65^{\prime \prime} \mathrm{E}$;

Lugavskoye - Krasnoyarsk territory, near Lugavskoye lake, 53०49'85"N, $91^{\circ} 88^{\prime} 2^{\prime \prime} \mathrm{E}$;

Mayna - Khakassia, Beya district, W. Sayan, close Mayna settlement, from Mayna to marble quarry road (6 km), mountain taiga $52^{\circ} 57^{\prime} 28^{\prime \prime} \mathrm{N}, 91^{\circ} 24^{\prime} 01^{\prime \prime} \mathrm{E}, \mathrm{h}=632 \mathrm{~m}$.;

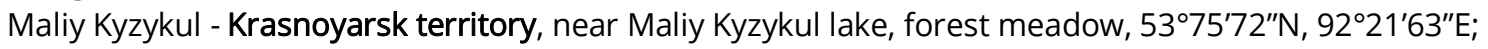

Manysh - Khakassia, W. Sayan, Tashtyp district, near Manysh river, mountain taiga meadow, 51 ${ }^{\circ} 52^{\prime} 36^{\prime \prime} \mathrm{N}, 8^{\circ} 48^{\prime} 8^{\prime \prime} \mathrm{E}$. $\mathrm{h}=1122 \mathrm{~m}$;

Minino - Krasnoyarsk territory, Krasnoyarsk vicinities, Minino village, river Karaul 'naya, 56 4'52.93" $\mathrm{N}$, 92²' $58.92 " \mathrm{E} ;$

Novoselovo - Krasnoyarsk territory, near Novoselovo village, 550'32.35"N, 9058'33.14"E;

Oglahty - Khakassia, Khakassky state National Reserve, Oglahty area, motley grass steppe, rocky hills. 53⒌'22" $\mathrm{N}$, $91^{\circ} 29^{\prime} 31^{\prime \prime} \mathrm{E}, \mathrm{h}=336 \mathrm{~m}$;

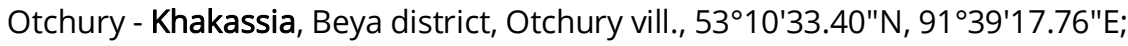

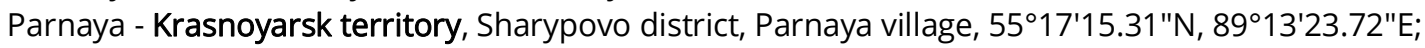

Podlunnaya - Khakassia, Kuznetsky Alatau mountains, Shira district, close Kommunar mine, Podlunnaya mountain, mountain taiga, subalpine meadow. 54 ${ }^{\circ} 18^{\prime} 36.61^{\prime \prime} \mathrm{N}, 89^{\circ} 13^{\prime} 42.79^{\prime \prime} \mathrm{E}, \mathrm{h}=1320 \mathrm{~m}$;

Podsinee - Khakassia, near Podsinee village, meadow on the river Enissey, 53⒍ $67^{\prime} 35^{\prime \prime} \mathrm{N}, 91^{\circ} 56^{\prime} 99^{\prime \prime} \mathrm{E}$;

Pugatshevo - Krasnoyarsk territory, Krasnoyarsk vicinities, Pugatshevo railway staition, 56 1'57.09"N, 92³7'38.35"E;

Pulankol - Khakassia, near Pulankol village, subtaiga zone, hills \& rocky, 53⒉ $28^{\prime} 33^{\prime \prime} \mathrm{N}, 90^{\circ} 25^{\prime} 52^{\prime \prime} \mathrm{E}$;

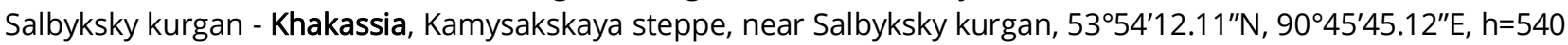

$\mathrm{m}$;

Sarala - Khakassia, Odzhonikidze district, Sarala, 545ำ'52.50"N, 89¹4'10.77"E;

Sayanogorsk - Khakassia, Beya district, Sayanogorsk town, 535' $47.93^{\prime \prime} \mathrm{N}, 9^{\circ}{ }^{\circ} 24^{\prime} 59.10^{\prime \prime} \mathrm{E} ;$

Saylig-Khem-Taiga - Khakassia, Tashtyp district, W. Sayan, Saylig-Khem-Taiga ridge, mountain tundra, h=2140m, $51^{\circ} 72^{\prime} 50^{\prime \prime} \mathrm{N}, 89^{\circ} 90^{\prime} 34^{\prime \prime} \mathrm{E} ;$

9131'49"E;

Serebryany Bor - Krasnoyarsk territory, Krasnoturansk district, Serebryany Bor camp, forest-steppe. $54^{\circ} 15^{\prime} 58^{\prime \prime} \mathrm{N}$, 
$89^{\circ} 94^{\prime} 09^{\prime \prime} \mathrm{E} ;$

Serebryany Klyuch - Khakassia, Tashtyp district, W. Sayan, A161rd. (215km), mountain taiga, h=978 m, 52³6'75"N,

Shira - Khakassia, Shira district, Kuznetsky Alatau foothills, 542ㄱ' $22.65^{\prime \prime} \mathrm{N}, 89^{\circ} 52^{\prime 2} 2.59^{\prime \prime} \mathrm{E}$;

Snezhnitsa - Krasnoyarsk territory, Krasnoyarsk vicinities, railway staition Snezhnitsa, 56 4'40.67"N, 92 204'55.58"E; Snow Leopard - Khakassia, Tashtyp district, W. Sayan, A161 road, "Snow Leopard" camp, high mountain taiga, mountain tundra, $\mathrm{h}=1350 \mathrm{~m}, 51^{\circ} 83^{\prime} 31^{\prime \prime} \mathrm{N}, 89^{\circ} 77^{\prime} 91^{\prime \prime} \mathrm{E}$;

Stolby - Krasnoyarsk territory, Krasnoyarsk vicinities, Stolby nature reserve, Zmeinyi hollow, 5555'22.15"N, 9250'54.30"E;

Sugesh - Khakassia, Tashtyp district, W. Sayan, Sugesh camp, mountain taiga, h=575m, 52 ${ }^{\circ} 71^{\prime} 71^{\prime \prime} \mathrm{N}, 89^{\circ} 92^{\prime} 09^{\prime \prime} \mathrm{E}$;

Tuim - Khakassia, Shira district, Tuim village, 5419'49.08"N, 8955'42.60"E;

Tyoia - Khakassia, W. Sayan, Abakansky ridge, near Vershina Tyoia, h=980m, 53⒉ $5^{\prime} 19^{\prime \prime} \mathrm{N}, 89^{\circ} 56^{\prime} 78^{\prime \prime} \mathrm{E}$;

Ust-Erba - Khakassia, near Ust-Erba village, steppe, 54⒋ $8^{\prime} 14^{\prime \prime} \mathrm{N}, 91^{\circ} 15^{\prime} 64^{\prime \prime} \mathrm{E}$;

Ust-Kaspa - Krasnoyarsk territory, Sayan foothills, near Ust-Kaspa railway station, rocky hill slope, Kyzyr river bank, taiga. $54^{\circ} 18^{\prime} 92^{\prime \prime} \mathrm{N}, 93^{\circ} 29^{\prime} 81^{\prime \prime} \mathrm{E}$;

Yezagash - Krasnoyarsk territory, Krasnoyarsk reservoir, Yezagash boundary, 55²7'9.73"N; 91 59'13.86"E;

Yuferovskoye - Khakassia, Kuznetsky Alatau, Sharypovo district, $3 \mathrm{~km} N$ of Yuferovskoye village, Bolshaya Sosnovka

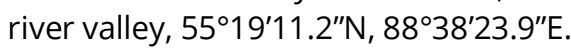

List of species reported from Khakassia and South of Krasnoyarsk Region for the first time is presented below. Here we included some species previously reported for the studied territory and confirmed by our collecting materials but not included in Region 22 (Catalogue, 2008).

\section{Family Zygaenidae}

3426 Zygaena purpuralis (Brünnich, 1763) - [Tshugunov, 1912; Djakonov, 1926; Wnukovsky, 1930]; Ust-Erba, 22.VII.2000, 1 specimen, R. Maksimov (RMA); Bidja, 19.VII.2015, 1 specimen, R. Maksimov (RMA); Novoselovo, 22.VII.2009, 1 specimen, M. Ivanov (MIK).

3434 Zygaena loti ([Denis \& Schiffermüller], 1775) - [Tshugunov, 1912]: Zygaena achilleae (Esper, 1780); Balgash, 16.VI.2011, 1 specimen, M. Ivanov (MIK).

Family Sesiidae

3470 Pennisetia hylaeiformis (Laspeyres, 1801) - Minino, daylight, 26.VII.2019, 1 specimen, M. Ivanov (MIK).

Family Cossidae

3610 Phragmataecia castanea (Hübner, 1790) - Maliy Kyzykul, at light, 25.VII.2015, 1 specimen; same locality, 10.VI.2017, 20', R. Maksimov (RMA).

Family Thyrididae

5218 Thyris fenestrella (Scopoli,1763) - [Kozhanchikov, 1923; Yanovsky, 1996]; Serebryany Klyuch, 01.Vl.2016, 5 specimens, S. Pokoreeva (RMA).

\section{Family Crambidae}

5893 Catoptria fenestratella (Caradja, 1928) - Lubimoe, 30.VI.2017, 1 specimen, R. Maksimov (RMA).

6046 Metaxmeste schrankiana (Hochhenwarth, 1785) - Saylig-Khem-Taiga, 05.VII.1999, 20', 19, R. Maksimov (RMA). 6028 Parapoynx stratiotata (Linnaeus, 1758) - Parnaya, Krasnoyarsk, 04.VIII.2010, 3 specimens, M. Ivanov (MIK).

\section{Family Drepanidae}

6495 Sabra harpagula (Esper, [1786]) - Maliy Kyzykul, at light, 10.VI.2017, 40,19, R. Maksimov (RMA); Krasnoyarsk, 11.VI.2015, 1 specimen, M. Ivanov (MIK).

\section{Family Geometridae}

6601 Selenia dentaria (Fabricius, 1775) - [Dyakonov, 1926]: Selenia bilunaria (Esper, 1795); Krasnoyarsk, 19.V.2015, 1 specimen, M. Ivanov (MIK); Tyoia, at light, 17.VI.2017, 20,19, R. Maksimov (RMA).

6679 Spilopera debilis (Butler,1878) - [Dyakonov, 1926]; Snezhnitsa, 18.VI.1989, 1 specimen, M. Ivanov (MIK); Ivanovskie lakes, 03.VII.2016, $40^{\circ}$, R. Maksimov (RMA); Tyoia, 30.VI.2018, 20',19, R. Maksimov (RMA).

6726 Macaria signaria (Hübner, 1809) - [Dyakonov, 1926; Yanovsky, 1996]; Snezhnitsa, 22.VI.2008, 1 specimen, M.

Ivanov (MIK); Podlunnaya, at light, 13.VII.2018, 20", 19, R. Maksimov (RMA); Tyoia, at light, 17.VI.2017, 10", R. Maksimov (RMA). 6738 Narraga fasciolaria (Hufnagel, 1767) - [Yakobson, 1901; Kozhanchikov, 1923; Djakonov, 1926; Wehrli, 1928];

Krasnoyarsk, 18.VI.2015, 1 specimen, M. Ivanov (MIK).

6739 Narraga tessularia (Metzner, 1845) - Minino, 30.V.2019, 10', M. Ivanov (MIK).

6792 Synopsia strictaria Lederer, 1853 - Krasnoyarsk, 24.VI.2008, 10', M. Ivanov (MIK).

6847 Pterygnophos ochrofasciata (Staudinger, 1896) - [Djakonov, 1926]: Gnophos ochrofasciata (Staudinger, 1896);

Chernoye Ozero, 06.VIII.2002, 1 specimen, E. Akulov (MIK).

6921 Alcis repandata (Linnaeus, 1761) - [Tshugunov, 1912; Djakonov, 1926; Wnukovsky, 1930]: Boarmia repandata

(L.); Krasnoyarsk, 8.VII.2017, 1 specimen, M. Ivanov (MIK); Saylig-Khem-Taiga, at light, 29.VI.2017, 3 ơ '0', R. Maksimov (RMA). 6952 Ascotis selenaria ([Denis et Schiffermüller], 1775) - [Djakonov, 1926]: Boarmia selenaria ([Denis et Schiffermüller], 1775); Lugavskoye, at light, 19.VI.2018, 20', R. Maksimov (RMA); Serebryany Bor, at light, 13.VII.2019, 19, R. Maksimov (RMA). 
6996 Biston strataria (Hufnagel, 1767) - Abakan, at light, 06.V.2017, 6o'; Bograd, at light, 11.V.2018, 30; Maliy Kyzykul, at light, 29.IV.2018, 30", R. Maksimov (RMA); Krasnoyarsk, 25.IV.2017, 10", M. Ivanov; Katcha, 14.V.2004, 10", I. Zakharzhevsky (MIK).

7003 Lycia zonaria (Denis \& Schiffermüller, 1775) - Lugavskoye, at light, 28.IV.2017, 2ơ; Bidja, at light, 08.V.2018, 10; Bograd, at light, 11.V.2018, 10', R. Maksimov (RMA).

7210 Holarctias rufinaria (Staudinger, 1861) - Ust-Erba, 13.VI.2015, 10', R. Maksimov (RMA).

7336 Costaconvexa polygrammata (Borkhausen, 1794) - Snow Leopard, at light, 30.VI.2017, 10", R. Maksimov (RMA).

7343 Catarhoe rubidata (Denis \& Schiffermüller, 1775) - [Djakonov,1926]: Cidaria rubidata (Denis \& Schiffermüller,

1775); Efremkino, at light, 11.VI.2017, 1 specimen, R. Maksimov (RMA).

7365 Xanthorhoe aridela (Prout, 1937) - Krasnoyarsk, 19.VI.2003, 1 specimen, E. Akulov (MIK).

7411 Earophila badiata ([Denis \& Schiffermüller], 1775) - [Djakonov, 1926]: Cidaria badiata ([Denis \& Schiffermüller], 1775); Krasnoyarsk, 24.IV.2015, 1 specimen, M. Ivanov (MIK); Katcha, 15.V.2009, 1 specimen, I. Zakharzhevsky (MIK); Pulankol, at light, 02.V.2018, 1 specimen; Bidja, at light, 08.V.2018, 1 specimen; Bograd, at light, 11.V.2018, 3 specimens, R. Maksimov (RMA).

7420 Pelurga taczanowskiaria (Oberthür, 1880) - Krasnoyarsk, 16.VI.2019, 3ơ; Pugatshevo, 01.VII.2017, 19, M. Ivanov (MIK);

7427 Larentia clavaria (Haworth,1809) - Efremkino, at light, 05.VIII.2017, 2 specimens, R. Maksimov (RMA).

7510 Heterothera serraria (Lienig, 1846) - [Kozhanchikov, 1923; Djakonov, 1926]: Cidaria serraria (Lienig, 1846); Katcha, 27.VI.2010, 1 specimen, I. Zakharzhevsky (MIK).

7688 Perizoma bifaciata (Haworth, 1809) - Chernoye Ozero, 25.VII.2002, 1 specimen, E. Akulov (MIK).

7722 Eupithecia abietaria (Goeze, 1781) - [Yakobson, 1901; Kozhanchikov, 1923; Djakonov, 1926]: Eupithecia abietaria analoga (Djakonov,1926); Krasnoyarsk, 21.VI.2018, 1 specimen, M. Ivanov (MIK).

7863 Eupithecia venosata (Fabricius, 1787) - Krasnoyarsk, 20.VI.2015, 1 specimen, M. Ivanov (MIK).

7930 Trichopteryx polycommata ([Denis \& Schiffermüller], 1775) - Nothopteryx polycommata grisea [Djakonov,

1926]; Pugatshevo, 01.V.2019, 10 specimens, M. Ivanov (MIK).

\section{Family Lasiocampidae}

7945 Poecilicampa populi (Linnaeus,1758) - Maliy Kyzykul, at light, 11.IX.2018, 20', R. Maksimov (RMA); Katcha, 20.IX.2010, 1 specimen; same locality,16.IX.2012, 1 specimen, M. Ivanov (MIK).

7986 Phyllodesma ilicifolium (Linnaeus, 1758) - Katcha, 15.VII.2010, 1 specimen, M. Ivanov (MIK).

7987 Phyllodesma japonicum (Leech, [1889]) - Gladenkaya, at light, 27.V.2018, 1 specimen, R. Maksimov; Efremkino,

at light, 16.VI.18, 1 specimen, R. Maksimov (RMA); Katcha, 4.VI.2000, 1 specimen; 30.V.2004 - 1 specimen, M. Ivanov (MIK). Ivanov (MIK). 8006 Odonestis pruni (Linnaeus, 1758) - Krasnoyarsk, 2.VII.2015, 1 specimen; Katcha, 15.VII.2008, 1 specimen, M.

\section{Family Sphingidae}

8101 Laothoe populi(Linnaeus,1758) - [Yanovsky, 1996]: Amorpha populi(Linnaeus,1758); Krasnoyarsk, 17.VI.1986, 10', I. Zakharzhevsky; Katcha, 13.VII.2007, 19, I. Zakharzhevsky; Snezhnitsa, 18.VI.2008, 10', M. Ivanov (MIK); Tyoia, 17.VI.2017, at light, 10', 1\%; Maliy Kyzykul, 16.VI.2018, at light, 20, R. Maksimov (RMA).

8136 Hyles chuvilini (Eitschberger, Danner \& Surholt, 1998); Krasnoye ozero, 20.VII.1993, 2 caterpillars, A. Chuvilin;

Bidja, 21.VI.2015, 10;; Oglahty, at light, 03.VII.2018, 4o', 19, R. Maksimov (RMA).

Family Notodontidae

8201 Notodonta tritophus ([Denis \& Schiffermüller], 1775) -Katcha, at light, 12.VI.1985, M. Ivanov (MIK); [Yanovsky,

1996].

8269 Odontosia sieversii (Ménétriés, 1856) - Yuferovskoye, at light, 30.IV.2018, 250", S.A. Knyazev (SKO); Maliy Kyzykul, at light. 29.IV.2018, 2 specimens, R. Maksimov (RMA); Pulankol, at light, 02.V.2018, 2 specimens, R. Maksimov (RMA), Krasnoyarsk, at light, 24.IV.2017, 1 specimen, M. Ivanov (MIK).

8296 Gluphisia crenata (Esper, 1785) - Podsinee, at light. 07.VI.2017, 20"0'; Lugavskoye, at light, 19.VI.2018, 2 specimens, R. Maksimov (RMA); Krasnoyarsk, at light, 2.VII.2018 - 1 specimen M. Ivanov (MIK); Katcha, 5.VII.2007, 1 specimen, M. Ivanov (MIK).

8301 Furcula furcula (Clerck, 1759) - Tyoia, at light, 17.VI.2017, 40', 3\%, R. Maksimov (RMA, SKO); Katch, 11.VI.2003,

1 specimen; Krasnoyarsk, 2.VII.2010, 1 specimen; Snezhnitsa, 8.VI.2011, 1 specimen, M. Ivanov (MIK).

8305 Cerura erminea (Esper, 1783) - [Kozhanchikov, 1923]: Dicranura erminea (Esper, 1783); Snezhnitsa, 15.VII.2010, 10, M. Ivanov; Krasnoyarsk, 18.VI.2015, 10, M. Ivanov; Katcha, 29.VII.2013, 10", I. Zakharzhevsky; same locality,

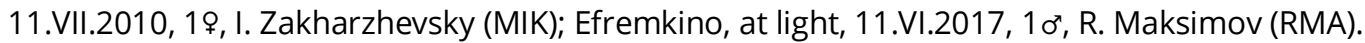

Family Noctuidae s.l.

8419 Nola karelica (Tengström, 1869) - Snow Leopard, at light, 30.VI.2017, 1 ơ, R. Maksimov (RMA).

8486 Earias clorana (Linnaeus, 1761) - [Kozhanchikov, 1925]; Katcha, 25.VI.2009, 1 speccimen, M. Ivanov (MIK);

Maliy Kyzykul, 16.VI.2019, at light, 1 specimen, R. Maksimov (RMA).

8521 Eublemma amasina (Eversmann, 1842) - [Kozhanchikov, 1923]: Porphyrinia amasina (Eversmann, 1842);

Katcha, 28.VI.2003, 1 specimen, M. Ivanov (MIK).

8537 Eublemma rosea (Hübner, 1790) - Chernoye Ozero, 16.VII.2018, 1 specimen, E. Akulov (MIK). 
8553 Trisateles emortualis ([Denis \& Schiffermüller], 1775) [Kozhanchikov, 1923]: Aethia emortualis ([Denis \& Schiffermüller], 1775); Krasnoyarsk, 20.VI.2015, 1 specimen, M. Ivanov (MIK).

8617 Simplicia rectalis (Eversmann, 1842) - [Tshugunov, 1913; Kozhanchikov, 1923]; Pugatshevo, 10.VII.2017, 1 specimen, M. Ivanov (MIK); Oglahty, at light, 03.VII.2018, 19, R. Maksimov (RMA).

8628 Zanclognatha tarsipennalis (Treitschke, 1835) - Pugatshevo, 07.VIII.2019, 1 specimen, M. Ivanov (MIK).

8648 Herminia tarsicrinalis (Knoch, 1782) - Pugatshevo, 12.VIII.2017, 1 specimen, M. Ivanov (MIK).

8692 Colobochyla salicalis ([Denis \& Schiffermüller], 1775) - Pugatshevo, 9.VIII.2018, 1 specimen, M. Ivanov (MIK).

8699 Laspeyria flexula ([Denis \& Schiffermüller], 1775) - Stolby, 8.IX.2016, 1 specimen, M. Ivanov (MIK).

8938 Macdunnoughia confusa (Stephens,1850) - [Kozhanchikov, 1923]: Phytometra confusa (Stephens, 1850);

Krasnoyarsk, 18.VIII.2014, 1 specimen, M. Ivanov (MIK); Ust-Kaspa, 02.VIII.2016, at light, 2 o"; Ergaky, 04.VI.2017, at light, 10";

Oglahty, 28.IV.2017, at light, 19; Maliy Kyzykul, 27.VIII.2016, at light, 1 ơ, R. Maksimov (RMA).

8950 Diachrysia stenochrysis (Warren, 1913) - Efremkino, at light, 05.VIII.2017, 20;; Maliy Kyzykul, 16.VI.2018, at

light, 10, 19, R. Maksimov (RMA); Krasnoyarsk, 24.VI.2008, 1 specimen, M. Ivanov (MIK).

8956 Euchalcia consona (Fabricius, 1787) - Khudonogovo, 20.VII.2006, 1 specimen, V. Golovizin (MIK); Oglahty, at

light, 07.VIII.2018, 2 specimens; Serebryany Bor, at light, 13.VII.2019, 2 specimens, R. Maksimov (RMA).

8968 Polychrysia splendida (Butler, 1878) - [Kozhanchikov, 1923]: Chrysoptera splendida (Butler, 1878);

Krasnoyarsk, 25.VII.2015, 1 specimen, M. Ivanov (MIK); Galdja, at light, 31.VII.2017, 2 specimens; Sugesh , at light,

11.VIII.2018, 2 specimens, R. Maksimov (RMA).

8970 Panchrysia deaurata (Esper, 1787) - [Kozhanchikov, 1923]: Phytometra deaurata (Esper, 1787); Krasnoyarsk,

2.VII.2010, 1 specimen, M. Ivanov (MIK); Podlunnaya, at light, 27.VII.2019, 1 specimen, R. Maksimov (RMA).

8981 Autographa bractea (Denis \& Schiffermüller, 1775) - [Zolotarenko, 1969]; Katcha, 3.VIII.2013, 1 specimen, I.

Zakharzhevsky (MIK); Podlunnaya, at light, 13.VII.2018, 2 specimens, R. Maksimov (RMA).

9008 Plusia festucae (Linnaeus, 1758) - [Kozhanchikov, 1928]: Phytometra festucae (Linnaeus, 1758); Khudonogovo,

20.VII.2006, 1 specimen, V. Golovizin (MIK); Efremkino, at light, 14.VII.2017, 1 specimen; Podlunnaya, at light, 27.VII.2019, 1

specimen, R. Maksimov (RMA).

9124 Acronicta alni (Linnaeus, 1767) - [Kozhanchikov, 1923; Zolotarenko, 1969]; Krasnoyarsk, 18.VI.2015, 1

specimen, M. Ivanov (MIK); Efremkino, at light, 16.VI.2018, 2 specimens; Tyoia, at light, 30.VI.2018, 2 specimens; Maliy

Kyzykul, 21.VII.2018, at light, 1 specimen, R. Maksimov (RMA).

9130 Acronicta concerpta Draudt, 1937 - Krasnoyarsk, 18.VI.2015, 1 specimen, M. Ivanov (MIK); Tyoya, at light,

17.VI.2017, 19 \& 30.VI.2018, 20;; Ergaky, 19.VI.2019, at light, 10", R. Maksimov (RMA);

9138 Acronicta lutea (Bremer et Grey, 1852) - [Kozhanchikov, 1928]: Chamaepora lutea (Bremer et Grey, 1852);

Sayanogorsk, 22.II.1982 (ex larva), 1 specimen, I. Zakharzhevsky (MIK); Efremkino, at light, 16.VI.2018, 1 specimen; Ergaky,

19.VI.2019, at light, 1 specimen, R. Maksimov (RMA).

9139 Acronicta major Bremer, 1861- Katcha, 1 specimen, I. Zakharzhevsky (MIK); Chernoye Ozero, 11.VII.2003, 1

specimen, E. Akulov (MIK).

9145 Acronicta rumicis (Linnaeus, 1758) - [Kozhanchikov, 1923]:Chamaepora rumicis (Linnaeus, 1758);

Krasnoyarsk, 18.VI.2015, 1 specimen, M. Ivanov (MIK); Tyoia, 22.VI.2019, at light, 1 specimen, R. Maksimov (RMA).

9153 Simyra nervosa ([Denis et Schiffermüller],1775) - [Kozhanchikov, 1923]; Krasnoyarsk, 5.VI.2007, 1 specimen,

M. Ivanov; Avraz, 05.VIII.2009, 1 specimen, I. Zakharzhevsy (MIK); Efremkino, at light, 14.VII.2017, 2 specimens, R. Maksimov (RMA).

9206 Cucullia absinthii (Linnaeus, 1761) - Khudonogovo, 20.VII.2006, 1 specimen, V. Golovizin (MIK).

9211 Cucullia asteris ([Denis et Schiffermüller], 1775) - [Kozhanchikov, 1923; Tshetverikov, 1925]; Galdja, at light,

31.VII.2017, 2 specimens; Oglahty, at light, 07.VIII.2018, 2 specimens, R. Maksimov (RMA).

9239 Cucullia pustulata Eversmann, 1842 - Snezhnitsa, 20.V.2000, 1 specimen, M. Ivanov (MIK); Lugavskoye, at light,

19.VI.2018, 1 specimen, R. Maksimov (RMA).

9268 Cucullia virgaureae Boisduval, 1840 - Katcha, 9.VII.2009, 1 specimen, M. Ivanov (MIK); Oglahty, at light,

07.VIII.2018, 1 specimen, R. Maksimov (RMA).

9281 Sympistis heliophila (Paykull, 1793) - Chernoye Ozero, 8.VII.2005, 1 specimen, E. Akulov (MIK).

9290 Oncocnemis senica (Eversmann, 1856) - Shira, 4.VIII.2009, 1 specimen; Krasnoyarsk, 25.VII.2013, 1 specimen,

M. Ivanov (MIK); Podlunnaya, at light, 04.VIII.2018, 1 specimen, R. Maksimov (RMA).

9303 Phidrimana amurensis (Staudinger, 1892) - Krasnoyarsk, 8.VIII.2018, 1 specimen, M. Ivanov (MIK).

9312 Amphipira pyramidea (Linnaeus,1758) - Maliy Kyzykul, at light, 12.VIII.2015, 1 specimen, R. Maksimov (RMA).

9314 Amphipyra sergei (Staudinger, 1888) - Katcha, 22.VII.2000, I. Zakharzhevsky (MIK).

Ivanov (MIK).

9316 Amphipyra tragopoginis (Clerck, 1759) - [Kozhanchikov, 1923]; Pugatshevo, 12.VIII.2017, 1 specimen, M.

9322 Brachionycha nubeculosa (Esper, 1785) - [Kozhanchikov, 1923]; Krasnoyarsk, 24.IV.2017, 1 specimen, M. Ivanov (MIK); Bidja, at light, 08.V.2018, 2 specimens; Gladenkaya, at light, 27.V.2018, 2 specimens; Oglahty, at light, 05.V.2019,

2 specimens, R. Maksimov (RMA).

9373 Helicoverpa armigera (Hübner, [1808]) - Pugatshevo, 1.X.2018, 1 specimen, M. Ivanov (MIK).

9385 Acosmetia caliginosa (Hübner, [1813]) - [Kozhanchikov, 1923]; Krasnoyarsk, 8.VI.2016, 1 specimen, M. Ivanov

(MIK). 
9442 Victrix umovii (Eversmann, 1846) - Katcha, 28.VI.2003, 1 specimen, I. Zakharzhevsky (MIK). 9444 Athaumasta expressa (Lederer, 1855) - Efremkino, 16.VI.2018, 10', R. Maksimov (RMA).

9447 Athaumasta siderigera (Christoph, 1893) - Efremkino, at light, 11.VI.2017, 10 ", R. Maksimov (RMA).

9466 Caradrina albina Eversmann, 1848 - Katcha, 14.IX.2002, 1 specimen, I. Zakharzhevsky (MIK).

9492 Hoplodrina blanda ([Denis \& Schiffermüller], 1775) - Krasnoyarsk, 24.VI.2015, 1 specimen, M. Ivanov (MIK).

9521 Athetis lepigone (Möschler, 1860) - Krasnoyarsk, 8.VI.2016, 1 specimen, M. Ivanov (MIK); Podlunnaya, at light,

13.VII.2018, 1 specimen, R. Maksimov (RMA).

9524 Athetis pallustris (Hübner, [1808]) - Katcha, 15.VI.2009, 1 specimen, I. Zakharzhevsky; Krasnoyarsk, 7.VI.2017,

1 specimen, M. Ivanov (MIK); Efremkino, at light, 16.VI.2018, 1 specimen, R. Maksimov (RMA).

9529 Ipimorpha contusa (Freyer, 1849) - [Kozhanchikov, 1928]; Krasnoyarsk, 24.VI.2015, 1 specimen; Stolby,

22.VII.2015, 1 specimen, M. Ivanov (MIK).

9541 Cosmia pyralina ([Denis \& Schiffermüller], 1775) - [Kozhanchikov, 1928]: Calymnia pyralina (View.);

Krasnoyarsk, 10.VII.2017, 1 specimen, M. Ivanov (MIK).

9605 Hyppa rectilinea (Esper,1788) - [Kozhanchikov, 1923]: Lithomoia rectilinea (Esper,1788); Krasnoyarsk,

25.VI.1985, 1 specimen, I. Zakharzhevsky; Snezhnitsa, 22.VI.2008, 1 specimen, M. Ivanov; Katcha, 24.VI.2005, 1 specimen, I.

Zakharzhevsky (MIK); Tyoia, 30.VI.2018, at light, 2 specimens; Podlunnaya, at light, 13.VII.2018, 2 specimens, R. Maksimov (RMA).

9608 Actinotia polyodon (Clerck, 1759) - [Kozhanchikov, 1923]; Katcha, 28.VI.2008, 1 specimen, I. Zakharzhevsky

(MIK); Efremkino, at light, 16.VI.2018, 1 specimen; Ergaky, 19.Vl.2019, at light, 1 specimen, R. Maksimov (RMA).

9659 Gortyna flavago ([Denis \& Schiffermüller], 1775) - Krasnoyarsk, 15.VIII.2017, 1 specimen, G. Kuleshov; Katcha,

27.VIII.2002, 1 specimen, I. Zakharzhevsky; Pugatshevo, 28.VIII.2018, 1 specimen, M. Ivanov (MIK).

9666 Hydraecia mongoliensis Urbahn, 1967 - Krasnoyarsk, 25.VII.2015, 1 specimen, M. Ivanov (MIK); Oglahty, at

light, 07.VIII.2018, 19, R. Maksimov (RMA).

9669 Hydraecia petasitis (Doubleday, 1847) - Maliy Kyzykul, at light, 12.VIII.2017, 2 specimens; Sugesh , at light,

19.VIII.2017, $1 \sigma^{\prime}, 19$, R. Maksimov (RMA).

R. Maksimov (RMA).

9692 Fabula zollikoferi (Freyer, 1836) - [Kozhantshikov, 1926]: Sidemia zollikoferi ab internigrata Warr.

9694 Rhizedra lutosa (Hübner, [1803]) - Khudonogovo, 20.VII.2006, 1 specimen, V. Golovizin; Chernoye Ozero,

28.VII.2005, 1 specimen, E. Akulov (MIK); Podsinee, at light. 23.IX.2018, 1o', 19; Oglahty, at light, 28.VIII.2019, 5o',19, R.

Maksimov (RMA).

9699 Nonagria typhae (Thunberg, 1784) - Maliy Kyzykul, at light, 27.VIII.2017, $10^{\prime}$ \& 11.IX.2018, 19 R. Maksimov

(RMA); Snezhnitsa, 21.VIII.2004, 1 specimen, M. Ivanov; Katcha, 14.IX.2012, 1 specimen, I. Zakharzhevsky (MIK).

9721 Hypocoena stigmatica (Eversmann, 1855) - [Kozhantshikov, 1925]: Coenobia stigmatica (Eversmann, 1855);

Yezagash, 5.VII.1984, 1 specimen, M. Ivanov (MIK).

9737 Capsula sparganii (Esper, 1790) - [Kozhantshikov, 1923]: Archanara sparganii (Esper, 1790); Parnaya,

4.VIII.2010, 1 specimen, M. Ivanov (MIK).

9739 Pabulatrix pabulatricula (Brahm, 1791) - [Kozhantshikov, 1923]: Parastichtis pabulatricula (Brahm, 1791);

Krasnoyarsk, 05.VIII.2010, 1 specimen; Sarala, 3.VIII.2008, 1 specimen, M. Ivanov (MIK).

9746 Apamea illyria (Freyer, 1846) - Snezhnitsa, 22.VI.2007, 1 specimen, M. Ivanov (MIK); Tyoia, 30.VI.2018, at light,

10'; Ergaky, 06.VII.2019, at light, 1 specimen, R. Maksimov (RMA).

9759 Apamea ingloria (Bang-Haas, 1912) - Saylig-Khem-Taiga, at light, 29.VI.2017, 5 specimens, R. Maksimov (RMA,

SKO).

9772 Lateroligia ophiogramma (Esper,1794) - [Kozhantshikov, 1928]: Parastichtis ophiogramma (Esper,1794);

Minino, 19.VII.2017, 1 specimen, M. Ivanov (MIK).

9793 Mesapamea secalis (Linnaeus, 1758) - [Kozhantshikov, 1923]: Parastichtis secalis rava (Haw.); Krasnoyarsk,

27.VII.2017, 1 specimen, M. Ivanov (MIK).

9806 Oligia latruncula ([Denis \& Schiffermüller], 1775) - Krasnoyarsk, 24.VI.2015, 1 specimen, M. Ivanov (MIK); Maliy

Kyzykul, 10.VIII.2019, at light, 1 specimen, R. Maksimov (RMA).

9809 Oligia strigilis (Linnaeus, 1758) - [Kozhantshikov, 1923]: Oligia strigilis aethiops (Haw.); Katcha, 15.VII.2012, 1

specimen, I. Zakharzhevsky (MIK).

9902 Lithophane lamda (Fabricius, 1787) - Krasnoyarsk, 15.V.2010, 1 specimen, M. Ivanov (MIK); Karynzul, at light,

08.V.2019, 1 specimen; Sugesh, at light, 31.VIII.2019, 1 specimen, R. Maksimov (RMA).

9926 Eupsilia transversa (Hufnagel, 1766) - [Kozhantshikov, 1923]: Eupsilia satellitia (L.); Krasnoyarsk, 24.IV.2015,

10'; same locality, 06.IX.2018 - 19, M. Ivanov (MIK); Podsinee, at light, 16.IV.2018, 1 specimen; Pulankol, at light, 21.IV.2018,

1 specimen; Mayna, at light, 18.IX.2019, 1 specimen, R. Maksimov (RMA).

9959 Antitype chi (Linnaeus, 1758) - [Kozhantshikov, 1923]; Katcha, 30.VIII.2011, 1 specimen, I. Zakharzhevsky;

Krasnoyarsk, 16.VIII.2015, 1 specimen, M. Ivanov (MIK).

10004 Orthosia cerasi (Fabricius, 1775) - Krasnoyarsk, 24.IV.2017, 1 specimen, M. Ivanov (MIK).

10015 Orthosia opima (Hübner, [1809]) - Bidja, at light, 08.V.18, 2, R. Maksimov (RMA); Krasnoyarsk, 16.IV.2011, 1

specimen, M. Ivanov (MIK).

10017 Orthosia populeti (Fabricius, 1775) - Katcha, 25.V.2009, 1 specimen, I. Zakharzhevsky (MIK). 
10023 Anorthoa munda ([Denis et Schiffermuller], 1775) - Lugavskoye, at light, 26.IV.2018, 3 specimens, R. Maksimov (RMA); Abakan, at light, 06.V.2017, 1 specimen, R. Maksimov (RMA); Bidja, at light, 08.V.2018, 3 specimens, R. Maksimov (RMA); Bograd, at light, 11.V.2018, 7 specimens, R. Maksimov (RMA); Yuferovskoye, at light, 30.IV.2018, 4 specimens, S.A. Knyazev (SKO); Krasnoyarsk, 13.IV.2011, 1 specimen, M. Ivanov (MIK).

10042 Cerapteryx graminis (Linnaeus, 1758) - Salbyksky kurgan, at light, 18.VII.2018, 1 specimen; Efremkino, 15.VII.2017, 1 ơ; Tyoia, at light, 29.VII.2017, 1 specimen, R. Maksimov (RMA); Stolby, 22.VII.2015, 1 specimen; Tuim, 2.VIII.2008, 1 specimen, M. Ivanov (MIK).

10050 Anarta imperspicua Hacker, 1998 - Saylig-Khem-Taiga, at light, 29.VI.2017, 3 specimens; Balankul, at light, 08.VI.2018, 2 specimens; Efremkino, at light, 16.VI.18, 1 specimen; Bidja, at light, 26.VI.18, 3 specimens, R. Maksimov (RMA); Krasnoyarsk, 2.VI.2011, 1 specimen, M. Ivanov (MIK).

10072 Polia conspicua (A.Bang-Haas, 1912) - Saylig-Khem-Taiga, at light, 29.VI.2017, 4 specimens; Podlunnaya, at

light, 13.VII.2018, 2 specimens; Gladenkaya, at light, 10.VII.2018, 2 specimens, R. Maksimov (RMA, SKO). 10077 Polia malchani (Draudt, 1934) - Ergaky, at light, 11.VI.2018, 2 specimens; same place, at light, 19.VI.2019, 1 specimen, R. Maksimov (RMA).

10117 Hada plebeja(Linnaeus,1761) - [Kozhanchikov, 1923]: Harmodia nana(Rott.); Ergaki, 17.VII.2009, 1 specimen, M. Ivanov; Krasnoyarsk, 25.VI.2008, 1 specimen, M. Ivanov (MIK); Snow Leopard, at light, 02.VI.2016, 1 \%; Saylig-Khem-Taiga, at light, 29.VI.2017, 2 o', R. Maksimov (RMA).

10136 Sideridis turbida (Esper, 1790) - [Kozhanchikov, 1923]: Trichoclea albicolon Hübner, [1813]; Shira, 6.VIII.2009, 1 specimen, M. Ivanov; Krasnoyarsk, 12.VI.2003, 1 specimen, E. Akulov (MIK); Efremkino, at light, 16.VI.2018, 2 specimens; Oglahty, at light, 03.VII.2018, 1 specimen, R. Maksimov (RMA).

10154 Conisania suaveola (Draudt, 1950) - Krasnoyarsk, 29.V.2001, 10", E. Akulov (MIK).

10158 Hecatera bicolorata (Hufnagel, 1766) - [Tshugunov, 1913]: Larentia bicolorata Hufn.; [Kozhantshikov, 1923]:

Polia serena leuconota (Ev.); Krasnoyarsk, 20.VI.2015, 1 specimen, M. Ivanov; Katcha, 18.VI.2008, 1 specimen, I. Zakharzhevsky (MIK).

10160 Hecatera dysodea ([Denis \& Schiffermüller], 1775) - Krasnoyarsk, 18.Vl.2007, 1 specimen; same locality,

28.VI.2018, 1 specimen, M. Ivanov; Khudonogovo, 20.VII.2006, 1 specimen, V. Golovizin (MIK). 10167 Hadena albimacula (Borkhausen, 1792) - [Kozhanchikov, 1923]: Harmodia albimacula (Borkhausen, 1792);

Efremkino, at light, 05.VIII.2017, 1 specimen, R. Maksimov (RMA).

10185 Hadena perplexa ([Denis \& Schiffermüller], 1775) - Oglahty, at light. 07.VIII.2018, 2 specimens, R. Maksimov

(RMA); Krasnoyarsk, 18.VI.2015, 1 specimen, M. Ivanov (MIK).

10217 Mythimna opaca (Staudinger, 1900) - Podsinee, at light, 22.V.2017, 2 specimens; Efremkino, at light,

11.VI.2017, 4 specimens; same locality, at light, 15.VII.2017, 1 specimen, R. Maksimov (RMA, SKO); Krasnoyarsk, 7.VII.2011, 1 specimen, M. Ivanov (MIK).

10221 Mythimna pudorina ([Denis \& Schiffermüller], 1775) - Katcha, 23.VII.2007, 1 specimen, I. Zakharzhevsky

(MIK).

10236 Leucania obsoleta (Hübner, 1803) - Podsinee, at light, 07.VI.2017, 10, R. Maksimov (RMA).

10242 Lasionycta alpicola Lafontaine \& Kononenko, 1988 - Ergaki, 17.VII.2009, 1 specimen, M. Ivanov (MIK); Saylig-

Khem-Taiga, at light, 01.VII.2017, 1 specimen; Manysh, at light. 25.VIII.2018, 1 specimen; Ergaky, 19.VII.2019, at light, 3 specimens, R. Maksimov (RMA);

10246 Lasionycta hampsoni Varga, 1974 - Saylig-Khem-Taiga, at light, 29.VI.2017, 6 specimens, R. Maksimov (RMA,

SKO).

10247 Lasionycta hospita A.Bang-Haas, 1912 - [Kozhantshikov, 1923; Filipyev,1925]: Lasionycta ardua

(Filipyev,1925); Saylig-Khem-Taiga, at light, 29.VI.2017, 1 specimen, R. Maksimov (RMA).

10250 Lasionycta leucocycla (Staudinger, 1857) - Saylig-Khem-Taiga, at light, 30.VI.2017, 1 specimen; Efremkino,

16.VI.2018, 1 specimen; Efremkino, at light, 16.VI.2018, 1 specimen, R. Maksimov (RMA).

10252 Lasionycta proxima (Hübner, 1809) - [Kozhantshikov, 1925]: Polia proxima extensa (Ev.); Snow Leopard, at

light, 30.VI.2017, 1 specimen; Efremkino, at light, 14.VII.2017, 1 specimen; same locality, at light, 05.VIII.2017, 1 specimen; Ergaky, 19.VII.2019, at light, 3 specimens, R. Maksimov (RMA); Krasnoyarsk, 2.VII.2010, 1 specimen; Tuim, 6.VIII.2009, 1 specimen, M. Ivanov (MIK).

10307 Dichagyris vallesiaca (Boisduval, 1837) - Oglahty, at light. 07.VIII.2018, 2 specimens, R. Maksimov (RMA). 10312 Euxoa aquilina ([Denis \& Schiffermüller], 1775) - Novoselovo, 28.VII.2009, 1 specimen, M. Ivanov (MIK).

10330 Euxoa eruta (Hübner, [1827]) - Shira, 4.VIII.2009, 1 specimen, M. Ivanov (MIK).

10353 Euxoa sibirica (Boisduval, 1832) - [Kozhantshikov, 1927]: Rhyacia sibirica (Boisduval, 1832); Otchury,

01.VIII.2009, 1 specimen; Krasnoyarsk, 07.VIII.2015, 1 specimen, M. Ivanov (MIK).

10407 Diarsia mendica (Fabricius, 1775) - Katcha, 13.VII.2010, 10, I. Zakharzhevsky (MIK).

10452 Chersotis cuprea ([Denis \& Schiffermüller], 1775) - Ingol ', 3.VIII.2010, 1 specimen; Krasnoyarsk, 4.VIII.2018,

1 specimen, M. Ivanov (MIK).

10472 Noctua interposita (Hübner, 1790) - Krasnoyarsk, 20.VIII.2015, 1 specimen, M. Ivanov (MIK); Saylig-Khem-

Taiga, at light, 29.VI.2017, 2 specimens; Gladenykaya, at light, 10.VII.2018, 3 specimens; Salbyksky kurgan, at light,

18.VII.2018, 2 specimens; Oglahty, at light, 03.VII.2018, 3 specimens, R. Maksimov (RMA).

10504 Xestia albonigra (Kononenko, 1981) - Katcha, 24.VII.2010, 10' I. Zakharzhevsky (MIK). 
10505 Xestia albuncula (Eversmann, 1851) - Gladenykaya, at light, 10.VII.2018, 4 specimens; same locality, at light, 28.VII.2018, 4 specimens; Podlunnaya, at light, 13.VII.2018, 2 specimens, R. Maksimov (RMA).

10508 Xestia atrata (Morrison, 1874) - Podlunnaya, at light, 13.VII.2018, 1 specimen; Gladenykaya, at light, 10.VII.2018, 2 specimens, R. Maksimov (RMA).

10510 Xestia banghaasi (Corti \& Draudt, 1933) - Podlunnaya, at light, 13.VII.2018, 6 specimens, R. Maksimov (RMA). 10550 Xestia sincera (Herrich-Schäffer, 1851) - Podlunnaya, at light, 13.VII.2018, 1 specimen, R. Maksimov (RMA).

10554 Xestia subgrisea (Staudinger, 1897) - Tyoia, at light, 29.VII.2017, 1 specimen, R. Maksimov (RMA); Katcha, 10.VIII.2011, 10', I. Zakharzhevsky (MIK).

10596 Naenia typica (Linnaeus, 1758) - Katcha, 1.IX.2000, 10", I. Zakharzhevsky (MIK).

Family Arctiidae

10651 Acerbia alpina (Quensel, 1802) - Saylig-Khem-Taiga, 18.VI.2000, 10', R. Maksimov (RMA). The species has been marked for 22-nd region as requiring confirmation in the Catalogue of the Lepidoptera of Russia [2008]. During the years of observation, we have collected only one specimen. It was caught in the daytime on the stones, in high mountain tundra. However, we confirm the presence of this species in Khakassia.

10788 Manulea complana (Linnaeus, 1758) - Pugatshevo, 10.VII.2017, M. Ivanov (MIK); Saylig-Khem-Taiga, at light, 28.VII.2002, 1 specimen; same locality, at light, 30.VI.2017, 1 specimen; Gladenykaya, at light, 28.VII.2018, 2 specimens; same locality, at light, 07.VIII.2019, 3 specimens, R. Maksimov (RMA).

\section{Discussion}

Thus, we reported 147 species from Khakassia and South of Krasnoyarsk region for the first time. Though some species were presented in publications earlier, however they were not related to 22 Region in Catalogue (2008). The main part of the material was collected during the period from 1999 to 2018 . The great share of the reported species is fairly common and sometimes attracted to the lights in large numbers. We suggested that the regional list of Heterocera of Khakassia and South of the Krasnoyarsk region should be considerably revised. Our research and observations will be continued and expanded to the new localities during the forthcoming field seasons. The most part of the specimens mentioned in the text are presented on the web-site "The Nature of South Siberia" by the following link: www.ermak24.com/animalia-insecta.html/.

\section{Acknowledgements}

We thanked our wives Svetlana Pokoreeva (Abakan) and Helen Knyazeva (Omsk) for invaluable help and understanding during all the stages of our work; E. Akulov, S.M. Loshev, Uchevatov I.B., V. Golovizin, G.V. Kuleshov, and I.S. Zakharzhevsky (Krasnoyarsk) for the collected materials they provided for our study. We are also thankful to Dr. V.V. Shurkina (Abakan), Deputy Director of Research State Natural Reserve "Khakassky", Director \& Deputy Director of Research Ergaki Nature Park I.V. Gryazin and Prof. N.V. Stepanov (Krasnoyarsk) for their support during our research in these reserves. The first author acknowledged Dr. T.A. Maksimova (Abakan) for generous field assistance during the trip.

\section{References}

Annotated catalogue of the insects of Russian Far East. Volume II. Lepidoptera. 2016. Vladivostok: Dalnauka. (In Russian). Catalogue of the Lepidoptera of Russia. 2008. SYu. Sinev (Ed.). Saint Petersburg-Moscow: KMK Scientific Press Ltd.

Djakonov AM. 1926. Zur Kentniss der Geometriden Fauna des Minussinsk Bezirks. Jarbuch des Martjanovischen Staatsmuseums in Minussinsk (Siberien) IV(I): 1-78. (In Russian).

Djakonov AM. 1927. Berichtigungen und Zusatsze zur Geometriden Fauna des Minussinsk Bezirks. Jarbuch des Martjanovischen Staatsmuseums in Minussinsk (Siberien) V(I): 54-58. (In Russian).

Filipiev NN. 1924a. Microheterocera des Minussinsk Bezirks. Jarbuch des Martjanovischen Staatsmuseums in Minussinsk (Siberien) II(III): 1-44. (In Russian).

Filipiev NN. 1924b. Microheterocera des Minussinsk Bezirks. Nachtrag 1. Jarbuch des Martjanovischen Staatsmuseums in Minussinsk (Siberien) II(III): 45-61. (In Russian).

Filipiev NN. 1925. Neue und venig bekannte Noctuiden aus dem Minussinsk Bezirks. Jarbuch des Martjanovischen Staatsmuseums in Minussinsk (Siberien) III(I): 59-67. (In Russian).

Filipiev NN. 1927. Microheterocera des Minussinsk Bezirks. Nachtrag 2. Jarbuch des Martjanovischen Staatsmuseums in Minussinsk (Siberien) V(I): 1-32. (In Russian).

Filipjev NN. 1930. Lepidopterologische Notizen. V. Microheterocera von Munku-Sardyk (Ost-Sajan-Geberige) Annuare du Musee Zoologique. Academie des Sciences URSS XXX: 1-15.

Koshantshikov WD. 1923. Materialjen zur Macrolepidopteren fauna des Minussinsk Bezirks. (Sibirien, lenissei Gov.) Jarbuch des Martjanovischen Staatsmuseums in Minussinsk (Siberien) I(I), I-VII:1-50. (In Russian).

Koshantshikov WD. 1925. Materialjen zur Macrolepidopteren fauna des Minussinsk Bezirks III. Jarbuch des Martjanovischen Staatsmuseums in Minussinsk (Siberien) III(I): 68-82. (In Russian). 
Koshantshikov WD. 1926. Materialjen zur Macrolepidopteren fauna des Minussinsk Bezirks IV. Jarbuch des Martjanovischen Staatsmuseums in Minussinsk (Siberien) IV(I): 79-82. (In Russian).

Koshantshikov WD. 1927. Materialjen zur Macrolepidopteren fauna des Minussinsk Bezirks V. Jarbuch des Martjanovischen Staatsmuseums in Minussinsk (Siberien) V(I): 51-52. (In Russian).

Koshantshikov WD. 1928. Materialjen zur Macrolepidopteren fauna des Minussinsk Bezirks VI. Jarbuch des Martjanovischen Staatsmuseums in Minussinsk (Siberien) VI(I): 64-82. (In Russian).

Korshunov YP, Viidalepp JR. 1980. Pyadenitsy (Lepidoptera, Geometridae) Khakassii. Soobshenie 1. Fauna \& ecology rastitelnoyadnyh \& hyshnych nasekomych Sibiry. Novosibirsk: Nauka. Sibirskoye otdelenye. (In Russian).

Korshunov YP, Viidalepp JR. 1982. Pyadenitsy (Lepidoptera, Geometridae) Khakassii. Soobshenie 2. Fauna \& ecology rastitelnoyadnyh \& hyshnych nasekomych Sibiry. Novosibirsk: Nauka. Sibirskoye otdelenye. (In Russian).

Lostchev SM. 2015. Entomofauna zapovednika "Stolby". Trudy gosudarstvennogo zapovednika "Stolby" 21: 1-216. (In Russian).

Meinhard AA. 1912. Obzor entomologicheskih zborov, proizvedennych v Zapadnych Sayanach I na Yenissee s 5-go avgusta po 8-e sentyabria 1903 goda. Tomsk. (In Russian).

Pittaway AR. 1993. The Hawkmoths of the Western Palaearctic. Colchester: Harley Books.

Pittaway AR. [Internet]. 2019. Sphingidae of the Western Palaearctic (including Europe, North Africa, the Middle East, western Siberia and western Central Asia); [cited 2019 Nov 28]. Available from: http://tpittaway.tripod.com/sphinx/list.htm/.

Sushkin PP, Tshetverikov SS. 1907. Lepidoptera Minusinsk district, West Sayan \& West part Uryanhayskaya steppe. Russian Entomology Society works XXXVIII: 3-34. (In Russian).

Tshetverikov SS. 1925. Beitrage zur Systematik der Noctuiden des Minussinskischen Bezirks. Jarbuch des Martjanovischen Staatsmuseums in Minussinsk (Siberien) III(I): 53-58. (In Russian).

Tshugunov SM. 1912. Lepidopteres chassees en ete 1909 pres du lac Ingol dans le gouvernement Enisej. Revue Russe d`Entom XII(2): 216-219. (In Russian).

Tshugunov SM. 1913). Les Lepidopteres collectionnes pendant I`ete 1912 dans le district Minussinsk gouvernement Jenissejsk. Revue Russe d`Entom XIII(2): 360-367. (In Russian).

Viidalepp JR. 1996. Checklist of the Geometridae of the former USSR. Stenstrup: Apollo Books (In Russian).

Volynkin AV. 2012. Noctuidae of the Russian Altay (Lepidoptera) Proceedings of the Tigirek State Natural Reserve. V. Barnaul.

Wehrli E. 1928. Beitrag zur Geometriden fauna von Minussinsk Bezirks. Jarbuch des Martjanovischen Staatsmuseums in Minussinsk (Siberien) VI(I): 8-30.

Wnoukovsky WW. 1930. Revisia nekotorych rabot Meyngarda A.A. \& Chugunova S.M. po faune cheshuekrylych Altaya, Minusinskogo kraya, Sayan \& Zapadnoy Mongolii. Trudy Sibirskogo Instituta selskogo hozyaystve \& lesovodstva. p. 275-285. (In Russian).

Yakobson A. 1901. Poyezdka v Abakanskye gory. Trudy Russkogo entomologicheskogo obshestva XXXV(1-2): 5-14. (In Russian).

Yanovsky VM. 1996. Lesnaya entomofauna Sayano-Shushenskogo biosphernogo zapovednika. Krasnoyarsk. Institut lesa im. V.N. Sukatcheva SO RAN (In Russian).

Zolotarenko GS. 1969. K faune sovok (Lepidoptera, Noctuidae) Vostochnogo Sayana. Trudy gosudarstvennogo zapovednika "Stolby" 7: 204-219. (In Russian).

\section{Citation:}

Maksimov RE, Ivanov MA, Knyazev SA. 2019. New records of Heterocera (Insecta, Lepidoptera) from the Republic of Khakassia and South of Krasnoyarsk region (South Siberia, Russia). Acta Biologica Sibirica 5(4): 12-20.

Submitted: 12.10 .2019 . Accepted: 20.11.2019

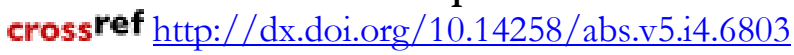

(C) 2019 by the authors. Submitted for possible open access publication under the terms and conditions of the Creative Commons Attribution (CC BY) license (http://creativecommons.org/licenses/by/4.0/). 